University of Nebraska - Lincoln DigitalCommons@University of Nebraska - Lincoln

2008

\title{
Liquid Space Lubricants Examined by Vibrational Microspectroscopy
}

Kenneth W. Street Jr.

Tribology and Surface Science Branch, NASA Glenn Research Center, Cleveland, $\mathrm{OH}$

Follow this and additional works at: http://digitalcommons.unl.edu/nasapub

Street, Kenneth W. Jr., "Liquid Space Lubricants Examined by Vibrational Microspectroscopy" (2008). NASA Publications. 194. http:// digitalcommons.unl.edu/nasapub/194

This Article is brought to you for free and open access by the National Aeronautics and Space Administration at DigitalCommons@University of Nebraska - Lincoln. It has been accepted for inclusion in NASA Publications by an authorized administrator of DigitalCommons@University of Nebraska - Lincoln. 


\title{
Liquid Space Lubricants Examined by Vibrational Microspectroscopy
}

\author{
Kenneth W. Street, Jr. \\ Tribology and Surface Science Branch, NASA Glenn Research Center, \\ Cleveland, $\mathrm{OH}$
}

\begin{abstract}
Considerable effort has been expended to develop liquid lubricants for satellites and space exploration vehicles. These lubricants must often perform under a range of harsh conditions such as vacuum, radiation, and temperature extremes while in orbit or in transit and in extremely dusty environments at destinations such as the moon and Mars. Historically, oil development was guided by terrestrial application, which did not provide sufficient space lubricants. Novel fluids such as perfluorinated polyethers provided some relief but are far from ideal. With each new fluid proposed to solve one problem, other problems have arisen. Much of the work performed at National Aeronautics and Space Adminstration's (NASA) Glenn Research Center, in elucidating mechanisms by which chemical degradation of space oils occur, has been done by infrared and Raman microspectroscopy, which this review details. Fundamental lubrication studies are presented as well as actual case studies, in which vibrational spectroscopy led to millions of dollars in savings and potentially prevented loss of mission.
\end{abstract}

Keywords: Vibrational spectoscopy, FTIR, Raman, space lubricants, tribology

\section{INTRODUCTION}

The future of space exploration will include missions to the moon and Mars. A typical mission scenario might include boosting exploration equipment into orbit, perhaps low earth orbit (LEO) for assembly, then continuing to a destination. Possible environmental exposure conditions while in LEO are

Received 23 November 2007; accepted 10 December 2007

Address correspondence to Kenneth W. Street, Jr., NASA GRC, MS 23-2, 21000 Brookpark Road, Cleveland, OH 44135. E-mail: kenneth.w.street@nasa.gov 
summarized in Table 1. Values were abstracted from LEO exposure of the Hubble space telescope between April 1990 and December 1993, and represent typical values that might be encountered (de Groh et al. 2001). Other exposure conditions and their effects on system failures have been documented (Fusaro and Khonsari 1992 and the databases referenced therein). Many of the values in Table 1 are dependent on the direction in which the surface is exposed with respect to the positions of the earth or the sun. For transport from LEO, conditions progressively change toward conditions experienced on the moon, with the possible exception of experiencing lower temperatures further from the sun. Conditions on destinations, our moon and Mars, are summarized in Table 2. The effect of these environments on space lubricants used for designing surface exploration vehicles is critical. A recent review by Jones and Jansen (Jones and Jansen 2005) presents an overview of the state of the art in space lubricants through January 2005.

In the early days of satellites, lubricants were not the failure points and the requirements for lubricating fluids were significantly fewer because satellites typically failed for other reasons. With the development of more robust hardware, the need for better lubricants became imperative because failures were, and in many cases still are, caused by failure of lubricated moving mechanical assemblies.

The first Apollo missions to include a vehicle to transport astronauts (Lunar Roving Vehicle (LRV) in Apollo 15 in 1971 and Apollo 17 in 1972) had specifications for operation in an environment of $\pm 120^{\circ} \mathrm{C}$ for up to two years. In order to expedite the delivery schedule, these requirements were eventually reduced to operation in $0-100^{\circ} \mathrm{C}$ for only a few hours. In order to provide adequate lubrication to the wheel drive unit (traction drive assembly) with lubricants available at the time, the units were hermetically sealed with a nitrogen atmosphere at approximately $50 \mathrm{kPa}$ (380 Torr or $\frac{1}{2}$ atmosphere) pressure. Two separate reports list the lubricant for the traction drive assembly as either Krytox ${ }^{\mathrm{TM}}$ 143AZ oil (Jones and Nola 1971) or Krytox $^{\text {TM }}$ 143AC oil (Cowart 1973), both of which are perfluorinated polyether (PFPE) oils. This author believes that Krytox ${ }^{\mathrm{TM}} 143 \mathrm{AC}$ was actually used. Other lubricating materials included Krytox ${ }^{\mathrm{TM}} 240 \mathrm{AC}$ grease

Table 1. Potential environmental conditions for low earth orbit

\begin{tabular}{lcc}
\hline Exposure & Range & Units \\
\hline Temperature & -100 to +50 & ${ }^{\circ} \mathrm{C}$ \\
X-Ray fluence $(1-8 \AA)$ & 40 & $\mathrm{~J} \mathrm{~m}^{-2} \mathrm{yr}^{-1}$ \\
Other electromagnetic radiation & $\mathrm{UV}, \mathrm{Vis}, \mathrm{IR}$ & \\
Electron fluence $(>40 \mathrm{keV})$ & $5.4 \times 10^{12}$ & $\mathrm{~cm}^{-2} \mathrm{yr}^{-1}$ \\
Proton fluence $(>40 \mathrm{keV})$ & $2.2 \times 10^{9}$ & $\mathrm{~cm}^{-2} \mathrm{yr}^{-1}$ \\
$\begin{array}{l}\text { Atomic oxygen fluence (primarily in ram } \\
\text { direction) }\end{array}$ & $8.6 \times 10^{19}$ & \\
\hline
\end{tabular}


Table 2. Potential environmental conditions for the moon and mars

\begin{tabular}{lcc}
\hline Exposure & Range & Units \\
\hline Moon & $1 \times 10^{-11}$ & \\
Vacuum & -170 to 110 (day cycle) & Torr \\
Temperature & Magnetic & ${ }^{\circ} \mathrm{C}$ \\
Dust & $\mathrm{X}$-Ray, $\gamma$-Rays, UV, Vis, IR & \\
Space radiations & 27.3 & day \\
Day cycle & & \\
Mars & 0.01 & $\mathrm{Atm}$ \\
Atmosphere $\left(\mathrm{CO}_{2}\right)$ & -143 (pole) to 27 (equator) & ${ }^{\circ} \mathrm{C}$ \\
Temperature & Electrostatic & \\
Dust & $50-100$ & $\mathrm{Micron}$ \\
Dust size & 24.6 & $\mathrm{hr}$ \\
Day cycle & $10-20$ & $\mathrm{~cm}$ \\
Dust height above surface & up to 15 & $\mathrm{~m} / \mathrm{s}$ \\
Wind velocity & 1 & $\mathrm{~m}$ \\
Permafrost depth $( \pm 40$ & Not evaluated & \\
$\quad$ degrees from equator) & Peroxides and superoxides & \\
Space radiation & & \\
Regolith oxidant components & &
\end{tabular}

in the traction drive and steering motors as well as silicone-based oil in the suspension damper. Dry lubrication was also employed. For comparison, data reported for the Russian robotic lunar explorers Lunokhods 1 and 2 indicate they were designed to provide 4 months of service. Two lubricants are listed: one characterized as an organo-silicon-based fluid (Lunokhod 1 2007) and the other undocumented (Lunokhod 2 2007). Current specifications for lubrication of NASA rovers and other surface system moving mechanical assemblies include a five-year lifetime. This will not be met by any of the liquid lubricants employed for Apollo missions because the use of hermetically sealed chambers containing artificial atmospheres is impractical for this time span in the lunar environment. Further, future missions will require longer traverses than a few miles (Morea 1993) in what is now known to be an abrasive environment created by fine particulate lunar regolith (Gaier 2007).

Simultaneous with the development of vacuum tribometers has been an effort to develop better space lubricants. New fluids have been developed and employed, such as the Pennzanes ${ }^{\circledR}$ multiply alkylated cyclopentanes (MAC). Other promising classes of oils that have been proposed and partially characterized include silahydrocarbons and ionic fluids. The ideal lubricant would perform under all of the extreme conditions outlined in Tables 1 and 2; however, many mechanisms will not experience all of these conditions at the same time and some conditions may never be experienced at all. For example: (1) A mechanism enclosed in a sealed housing may never experience 
high vacuum but on the moon could definitely be exposed to the full range of temperature extremes; (2) For devices with labyrinth seals, the exposure to vacuum will either change with time or additional hardware to repressurize the compartment will add complexity to the component. It would be of great benefit to have a large number of qualified lubricants on the shelf for various space conditions during the early stages of design for exploratory vehicles and stationary systems. Further efforts to develop space-qualified oils, additives, and greases to fill the gaps in current generation lubricants is an area that must be actively pursued in order to provide a better selection of lubricants for designing systems that will be employed in space, on the moon, and on Mars.

\section{Factors Influencing Choice of Lubricants}

The following section is included to provide some perspective to readers unfamiliar with the field of space tribology - the study of friction, wear, and lubrication—particularly for use in space (Fusaro and Khonsari 1992).

Weightlessness is a contributor to lubricant selection in that conventional pumping systems for lubricant replenishment are not feasible. Under these conditions, lubrication systems traditionally rely on surface tension to distribute lubricant; this is an important property of base oils in choosing an appropriate oil for a given application. In recent years several novel "pumping scenarios" have been proposed to deliver lubricant to starved mechanisms, although none have found widespread use (Glassow 1976; James 1977; Lowenthal et al. 1985; Marchetti et al. 2003). Greases are the primary choice for lubricating large mechanisms. On the moon and other planets this is not an issue-the issue is added complexity and the consequential increased weight associated with oil transport mechanisms and reservoirs.

Volatility is a primary cause of oil and additive loss under reduced pressure conditions. This property is directly related to vapor pressure, a value tedious to measure for space oils and additives. The most rapid loss occurs when the atmosphere over the bulk oil is reduced to "zero," or in other words an ultrahigh vacuum for a given use temperature. Consequently, the vapor pressure of an oil versus temperature is a major consideration in choosing an appropriate oil for space lubrication, and generally the lower the vapor pressure, the more attractive a lubricant is. In practice, evaporation rate is more useful information because it allows for direct determination of the amount of lubricant required over the lifetime of a given mission, though it is component geometry dependent.

Lubricant condensation may occur once molecules leave the site of application and redeposit themselves elsewhere. This can be a critical problem if the condensation occurs where it will damage or impede function of another component, for example, on lenses or optical windows. 
Thermal conductivity of the lubricant and tribocouple should also be considered. In vacuum, there is no gas convection mechanism for heat transport away from the site of lubrication, potentially leading to excessive heating, which can degrade the lubricant. Lower viscosity provides some relief because it allows circulation to remove and redistribute the heat, but in general, circulation is not considered a major pathway for cooling. Both lower viscosity, typically associated with lower molecular weight, and heating lead to undesirable higher vapor pressures.

Liquid lubricant viscosity and its variation with temperature are primary design considerations involving a number of trade-offs. In general, the higher the molecular weight, the greater the viscosity and the higher the temperature, the lower the viscosity. Whereas the former is often true, the latter is always true. As viscosity decreases, creep increases providing both a higher surface area for evaporative losses and a direct pathway for liquid migration from the application site. In terms of tribology, higher viscosities are beneficial in that elastohydrodynamic lubrication can occur at lower velocities thus alleviating the lubricant thickness required for good boundary lubrication. On the other hand, higher viscosity leads to higher torque in the lubricated device, resulting in higher power demand for operation.

Oxygen, readily available in air, serves as a reactant that enhances the friction and wear capabilities of various tribocouples by several mechanisms. First, oxygen reacts with many metals to form surface oxides, which have better friction characteristics than the bare metal contact. Second, oxygen is known to be a direct participant in the functioning of additives. Lack of oxygen must be considered when choosing a lubricant where no oxygen will be available to assist the lubricant. As a reactant, oxygen may also serve to degrade carbon-based lubricants during ground testing. Properties of lubricants for space considerations must be adequately evaluated for both atmospheric operation (ground testing) and space conditions (the actual application). Water is another reactant that is of considerable importance in the functioning of lubricants (Pepper 2005). When choosing a lubricant, the same considerations should be given to water as oxygen; however, the effect of moisture extending the lifetime of lubricants is as yet unexplained.

Radiation damage can be categorized by type of source: electromagnetic (photon) or particulate. On earth, the atmosphere shields some electromagnetic radiation such as ultraviolet but this shielding does not occur in space. Sources of electromagnetic radiation span the spectrum from ionizing highenergy gamma rays through the visible into the infrared (IR) region. Ionizing and ultraviolet radiation can promote direct degradation of lubricants through well-established photochemical pathways. Absorption of IR serves as a heat source resulting in thermal degradation pathways for the lubricant. Solar particulate radiation usually has a shallow penetration depth although these charged particles can do considerable damage to organic compounds. 
Most particulate radiation is easily shielded but is still a concern for exposed lubricants, and ultrahigh energy particles, so-called cosmic rays, are nearly unshieldable without the thick atmospheric protective layer enjoyed on Earth.

General debris should have no impact on lubricant performance in space. However, for crew exploration vehicles and rovers, surface dust from the regolith, which is readily kicked up, often sticks tenaciously to all surfaces it contacts (Gaier 2007; Landis 1998). Certain components of the lunar regolith are magnetic and both lunar and Martian soils are readily charged. This puts an additional requirement on lubrication of exposed components in that the lubricant should not act as a getter of particulate matter, which would jam a contact or generate excessive abrasive wear. The surface dust on the moon is composed of very fine structures (particles) from millennia of pulverization by impacts and, because there is no atmosphere or erosion mechanism for polishing, these dust particles also have sharp edges and points.

The chemistry of tribocouples, parts in relative moving contact, also greatly affects the performance of various lubricants. Certain elements, such as titanium in alloys, are notorious for decomposing perfluorinated polyether base fluids to form catalytic metal fluoride, which further increases the decomposition rate. Hence, choice of both construction materials and lubricant must be carefully considered during the design stage. The reverse effect has also been demonstrated - coating metal surfaces with various solids and surface treatments has been shown to increase lubricant lifetime. Additives have generally proven ineffective for a number of reasons, but the majority fails because the vapor pressures of the additives are greater than the vapor pressure of the base oil.

To evaluate which lubricants and tribocouples are best suited for space application, traditional tribometers have been adapted for vacuum operation. Tribometers (for example the vacuum 4-ball tribometer, a modification of the American Society for Testing and Materials, International (ASTM) 4-ball method) typically give wear information, coefficient of friction, and relative lifetime information. These experiments do not provide direct information about the mechanism of lubricant failure, which as a chemical process requires postmortem analysis of the tribocouples and lubricant residues. The use of surface analytical techniques has greatly enhanced the understanding of tribocouple chemistry and lubricant degradation. X-ray photoelectron spectroscopy (XPS) has been used to look at bearings after failure to identify surface species that may have contributed to failure. Though XPS gives limited chemical information, it was used to identify metal fluoride formation during degradation of PFPE lubricants. This analysis allowed researchers to test PFPE decomposition on metal fluorides to establish the catalytic behavior and degradation pathways associated with metal fluorides. However, XPS does not provide enough molecular structural information for the examination of the carbon-based liquid lubricants. To this end, a great deal of research at NASA's Glenn Research Center has been devoted to the use of vibrational spectroscopy to determine degradation pathways of base oils, reviewed here. 


\section{EXPERIMENTAL}

For perspective, Figs. 1a-c show example rigs used to acquire tribological data. While many types of tribometers exist, at our facility we have developed and primarily use Spiral Orbit Tribometers (SOT) 4-ball tribometers, and Pin on Disc (PoD) tribometers, Figs. 1a $-\mathrm{c}$ respectively, modified to work in high $\left(<10^{-6}\right.$ Torr $)$ to ultrahigh $\left(10^{-8}\right.$ Torr or lower $)$ vacuum. It should be emphasized that there are many varieties of tribometers but not all are adapted to ultrahigh vacuum, a criterion for doing space tribology. Simple blanketing of the sample with even research-grade inert gas has been brought into question due to trace contamination from water (Pepper 2005). Because of the ability to obtain relative lifetime data for lubricants in SOT, we have moved away from PoD testing in recent years. The SOT, in which a ball rolls between a fixed plate and a rotating plate, simulates an angular contact ball bearing. The load, often chosen to provide a mean Hertz stress of $1.5 \mathrm{GPa}$, is applied through the fixed plate. The combination of high load, moderate speed, and small amount of lubricant (typically $\sim 50 \mu \mathrm{g}$ ) allows the system to always operate in the boundary lubrication regime, where lubricant quickly degrades. The ball rolls and pivots in an ever increasing spiral; the track and the orbit are reestablished by the guide plate. The force the ball exerts on the guide plate determines the friction coefficient, since the ball is sliding between disks at the moment of measurement. The flat regions where the ball contacts both the guide plate and the stationary disc simultaneously are referred to as the scrub. From these figures it is obvious that the contact area where tribology occurs is very small. For example, the ball in the SOT is typically $12.7 \mathrm{~mm}$ diameter (although smaller balls have been used) and in the PoD the pins are shaped to resemble a $6.35 \mathrm{~mm}$ diameter ball, both of which have contact regions of only a few tens of square micrometers. In the four-ball rig, all balls are held rigidly in position and the load is applied vertically while rotating the top ball. After a fixed number of rotations, the experiment is stopped to
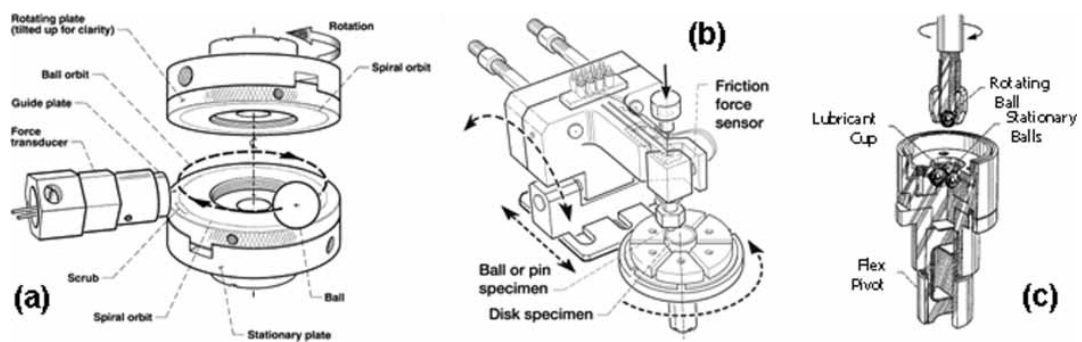

Figure 1. Schematic diagrams of tribometers adapted to vacuum conditions: (a) Spiral orbit tribometer (SOT); (b) Pin on disc tribometer (PoD); and (c) Four-ball wear tester. 
measure the wear scar dimensions on the balls. This process is repeated several times and the lost material is calculated for each step in the process. A plot of material loss versus the number of rotations allows computation of wear rate. In all of these experiments high quality tribocouples-metal tribological contacting surfaces often used in space bearings - are employed, such as 440C stainless steel hardened to 58-62 Rockwell C.

The use of conventional Fourier transform infrared (FTIR) and Raman spectrometers to characterize synthetic reaction products is a normal part of organic chemistry that will not be discussed, nor will their use for quality control quantification of additives to oils, another obvious use of these techniques. A more interesting use of the techniques is in the determination of reaction products after application of oils, which is referred to as postmortem analysis. Because the surface area of interest in postmortem work is typically small-tracks on the order of $0.1 \mathrm{~mm}$ across, or the curved surface of a ball bearing - the techniques suited for small area analysis have gained popularity. In our lab we use micro-FTIR and micro-Raman, which employ microscopes offering great advantage in analyzing small-spot areas, with Raman particularly well-suited. As a scattering technique, radiation is collected from almost any type of surface (curved or flat) with spot sizes down to the low micrometer dimension even though it does not provide the rich spectral data of IR. The FTIR microscope must either use a flat surface or the crown of a curved surface, as the radiation is directional and in addition must examine a much larger area, tens of micrometers diameter (Katon et al. 1980). In our instrument the standard aperture interrogates an area of 100 micrometers in diameter. This means that flat surfaces or the crown of a curved surface must be oriented normal to the microscope axis. Since a large amount of information is obtained from balls, particular attention to orientation is important, especially if grazing angle objectives are employed to enhance sensitivity (Pepper 1995).

\section{Lubricant Quantification in Studies by FTIR}

In earlier studies, thin lubricant layers were applied to PoD and SOT discs and thickness was quantified by direct reflectance micro-FTIR with grazing angle objectives to enhance sensitivity (Shogrin et al. 2003; Jones, Poslowski et al. 1999; Shogrin et al. 1996). Calibration was performed by establishing optical constants for Beer's law by measuring film thickness with ellipsometry in the visible wavelength range (Pepper 1995). This method of quantifying the amount of lubricant has several drawbacks for tribological use. In these studies, lubricant was applied as a thin uniform film to the discs. Consequently there was no method of quantifying the amount of lubricant consumed during the experiment. If the lubricant could be quantified, additional information about the relative lifetime of lubricants could be gained by normalizing the number of orbits at failure by the micrograms of lubricant consumed, or a normalized lifetime. 
Therefore, a method of metering a known amount of lubricant to the ball was devised and the amount of lubricant was quantified by gravimetry, a tedious measurement for a few micrograms of oil on a ball itself often exceeding $10 \mathrm{~g}$. In this method, the ball is magnetically held against a horizontally rotating shaft at one point, designated here as its north pole, and a measured volume of the lubricant solution diluted in solvent is applied to the rotating surface from a gas-tight microsyringe. After the solvent evaporates, the weight gained by the ball is determined by a microbalance with a resolution of \pm 2 microgram. The smallest weight of lubricant that can be reliably quantified in this manner is in the 10 microgram range.

A major limitation of infrared microspectroscopy arises when the coating thickness is not uniform over the surface and quantification is desired. Analysis thus requires spectral information from the entire surface or at least a statistically significant fraction thereof. However, to examine the surface of a $12.7 \mathrm{~mm}$ diameter ball bearing with a 100 micrometer diameter viewing objective, over $10^{6}$ spectra would be required and this is not feasible. Integration spheres have long been available as attachments for FTIR spectrometers. Although they are used traditionally for scattering transmission or reflectance measurements from materials and surface coatings, it is also possible to mount objects having symmetrical geometry, such as balls within integrating spheres, and to obtain spectra from significant portions of the complete surface, thus averaging over local heterogeneity (Street, Pepper et al. 2007).

Figure 2 shows a simplified schematic of the integration sphere with ball bearing sample. The intensity of the incoming beam is not uniform, but is a complex function of the spectrometer aperture and beam steering optics within the FTIR bench. For a flat sample at the reflectance port, the beam converges on the center of the sample, but for three-dimensional samples, the higher the sample, the further the center of the sample will be from the center of the beam such that the beam covers an estimated 20 to $50 \%$ of the ball's surface. Coverage depends primarily on (1) the aperture

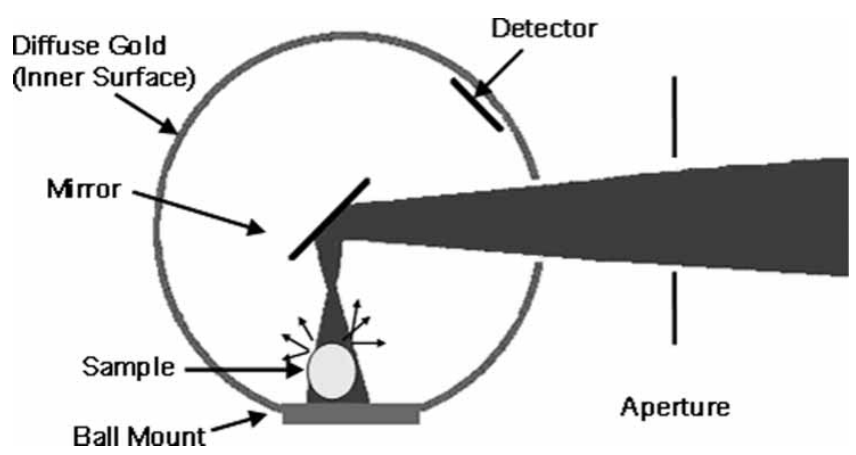

Figure 2. Schematic of integration sphere technique for examining ball bearings. 
setting, (2) the ball diameter, and (3) the positioning of the ball with respect to the center of the beam. Because of these uncertainties, five spectra (see Fig. 3) were obtained from each ball to provide information from the entire surface area. The positions investigated on the ball include the north and south pole and three evenly spaced positions about the equator. Optimization of other parameters is described in the paper. Figure 4 is a plot of the average absorbance for the C-H stretch bands at $2923 \mathrm{~cm}^{-1}$ for the five positions where the spectra were taken on different $12.7 \mathrm{~mm}$ balls versus the lubricant charge. Using this methodology it was determined that the limit of detection was significantly lower than by the gravimetric method. Additional benefit is derived by the incident IR beam striking the ball at oblique angles similar to the enhancement observed in grazing angle spectrophotometry. Calculations indicate a five-fold enhancement in absorbance over what would be predicted using two passes at normal incidence through the sample on the ball.

\section{Qualitative Information from Tribometer Studies}

Microscopes, both Raman and IR, are preferred for examining small areas on balls, discs, and guide plates, where residual used oil may be observed in droplets or for small damage features and so on. Microscopes also provide an interrogation area that is often sufficient to examine the centers of tracks on discs, residual material adjacent to tracks, and the scrub regions of discs and guide plates. During the course of IR examinations of balls it became evident that new techniques were needed to look at the condition of the entire ball to avoid being biased by events occurring on small areas. The introduction of

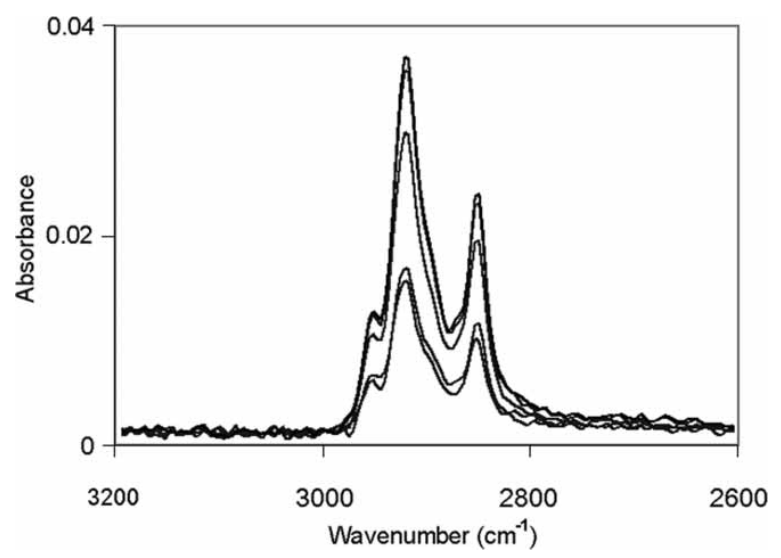

Figure 3. Spectra acquired from $50 \mu \mathrm{g}$ of Pennzane ${ }^{\circledR}$ on five different ball positions in an integrating sphere. 

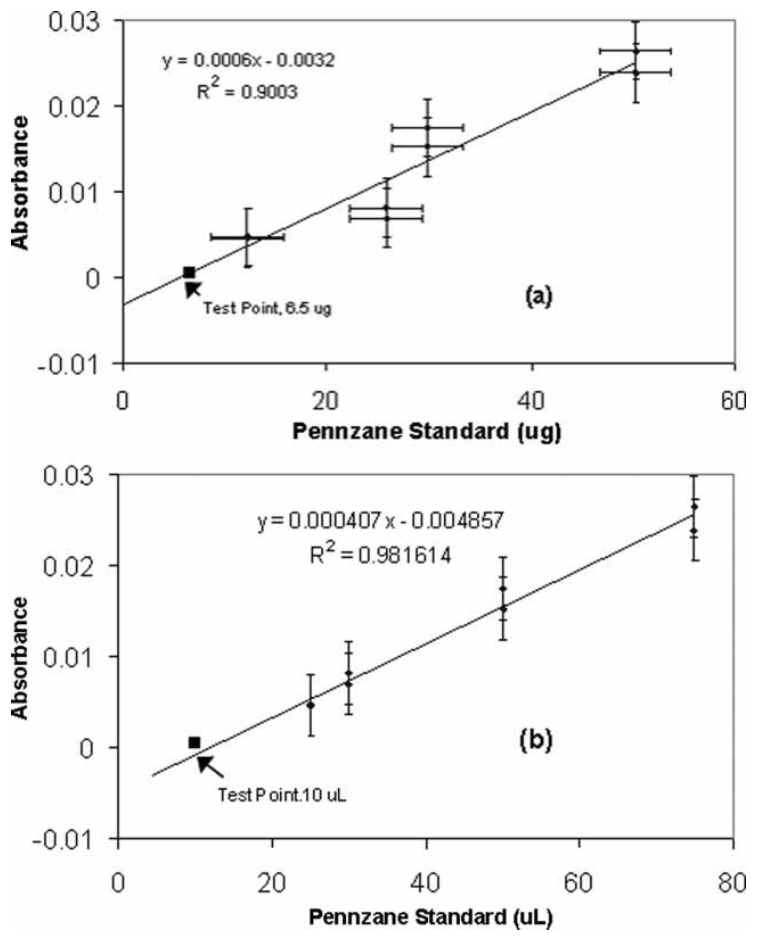

Figure 4. Plot of average absorbance for the $\mathrm{C}-\mathrm{H}$ stretch bands versus the lubricant charge: (a) lubricant charge in $\mu \mathrm{g}$ and (b) lubricant charge in $\mu \mathrm{L}$ of standard delivered.

the integrating sphere technique, as shown in Fig. 2, has greatly improved our ability to acquire information quickly. The technique, originally developed to quantify lubricant coatings applied to balls prior to testing at loadings below those readily quantifiable by gravimetry, has demonstrated utility in postmortem analysis. Figure 5 clearly shows that a significant amount of material is left on a ball after failure. In this particular case the residual material was freely washed from the ball, indicating that it was not polymerized during tribology and for some reason failure occurred prior to its consumption.

\section{RESULTS AND DISCUSSION}

\section{Properties of Ideal Lubricants}

As a consequence of the preceding discussion, there are some desirable features that should be considered when choosing a lubricant for nonterrestrial applications. Aside from the obvious friction and wear properties of the lubricant and tribological surface combinations, the most important 


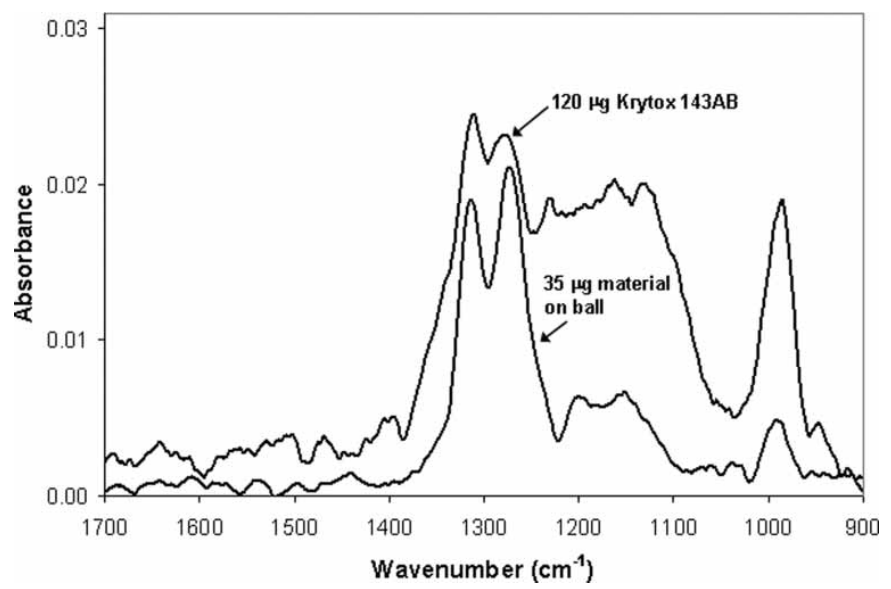

Figure 5. Spectra of ball lubricated with Krytox $^{\mathrm{TM}} 143 \mathrm{AB}$ and postmortem ball from SOT run with the same lubricant.

consideration is that the base oil survives launch, travel through space, and the destination site environment. Low vapor pressures are a primary consideration when prolonged exposure to vacuum is a part of the lifetime. The oil must remain liquid over a wide temperature range while providing adequate viscosity characteristics. At the low temperature extreme the ability of oil to perform is governed by its pour point, the temperature at which the oil will no longer flow, which is always higher than the melting point. Viscosity index, a measure of viscosity as a function of temperature, is used to rank performance, the higher index oils being preferred. It should be thermally stable and resistant to attack from oxygen, both molecular and atomic, and radiation tolerant. It should be a reasonable thermal transport medium. The oil should also be capable of being formulated with additives to provide good extreme pressure lubrication and for making of greases.

A large number of base oils have been developed over the past four decades. These include hydrocarbon (HC) and perfluoropolyalkylether (PFPE) materials summarized in Table 3. Mineral oil-based fluids, esters, and silicone oil are old technology materials still in use today for niche applications. Many of the mineral oil types are no longer manufactured and users have resorted to stockpiling to ensure future supplies. The earlier esters are no longer in production; however, a few low volatility neopentylpolyol esters are still marketed, such as UC4 and UC9 (Nye Lubricants 2007). All of these contain trixylenyl phosphate additive, which has a high vapor pressure. The silicone-based materials are poor boundary lubricants and tend to degrade into abrasive by-products.

Additives require either solubility in the base oil or the ability to form a long-term stable emulsion or dispersion. Additives are required for enhancing base oil properties, such as for extreme wear, oxidation, or poor boundary 
Table 3. Base oils for space lubrication

\begin{tabular}{|c|c|c|c|}
\hline Base fluid & Benefits & Drawbacks & Examples \\
\hline \multicolumn{4}{|l|}{ Hydrocarbon based } \\
\hline Mineral Oil & $\begin{array}{l}\text { Good boundary lubricants, } \\
\text { Easily formulated }\end{array}$ & $\begin{array}{l}\text { Requires additives, } \\
\text { high vapor pressure }\end{array}$ & Apeizon $\mathrm{C}$ \\
\hline Esters & Good boundary lubricants & Modest vapor pressure & \multirow{7}{*}{$\begin{array}{l}\mathrm{UC}_{4}, \mathrm{UC}_{7}, \mathrm{UC}_{9} \\
\text { Oils } 132,182 \text { and } 186 \\
\text { Pennzane }\end{array}$} \\
\hline Polyalphaolefin & Good boundary lubricants & Modest vapor pressure & \\
\hline Multiply & Good boundary lubricant & Higher vapor pressure than PFPE & \\
\hline Alkylated & Easily formulated & Lower viscosity than silahydrocarbons at $20^{\circ} \mathrm{C}$ & \\
\hline Cyclopentanes & Good Chemical stability & & \\
\hline \multirow[t]{2}{*}{ Ionic Liquids } & Low vapor pressures & Not fully developed & \\
\hline & Good friction coefficients & Many are proprietary formulations & \\
\hline \multicolumn{4}{|l|}{$\begin{array}{l}\text { Silicon containing } \\
\text { hydrocarbon based }\end{array}$} \\
\hline \multirow[t]{2}{*}{ Silicone oil } & Low vapor pressure & Poor boundary lubrication & \multirow[t]{2}{*}{ Versilube F-50 } \\
\hline & Low pour point & Abrasive decomposition products & \\
\hline \multirow[t]{2}{*}{ Silahydrocarbons } & Good boundary lubrication & Higher vapor pressure than PFPE & \multirow[t]{2}{*}{$\begin{array}{l}\text { Tri-, tetra- \& penta- } \\
\text { silahydrocarbons }\end{array}$} \\
\hline & Low volatility & & \\
\hline \multicolumn{4}{|l|}{ Non-hydrocarbon based } \\
\hline \multirow[t]{3}{*}{ Perfluoropolyether } & Good chemical \& thermal stability & Poor Boundary lubrication & \multirow{3}{*}{$\begin{array}{l}\text { Krytox }^{\mathrm{TM}}, \text { Fomblin, } \\
\text { Demnum }\end{array}$} \\
\hline & High viscosity index & Traditional additives not soluble & \\
\hline & Low pour points & & \\
\hline
\end{tabular}


lubrication. For these specific purposes, the additive is best dissolved in the base oil. To produce greases, soaps have been employed. These are soluble in the base oil and provide the desired degree of thickening to prevent creep and other problems associated with migration of oil from the site of lubrication. Solid insoluble additives like Teflon ${ }^{\circledR}$ have long been used to thicken PFPE-based greases. Solid additives need to disperse well and not separate from the base oil over time, while still releasing oil to the site of application to maintain lubrication.

Soluble hydrocarbon oil additives have been around for decades and are well-established for terrestrial operation. Many conventional additives like tricresyl phosphate have been directly used in space lubricants with mixed success. These additives are readily soluble in hydrocarbon space oils, but have significantly higher vapor pressures than base oils. Consequently, they evaporate quickly in vacuum, resulting in lubrication with properties of the base oils. Hydrocarbon base oils tend to provide better boundary lubrication than PFPE-based oils, but they still require antioxidants, anticorrosion, and antiwear additives yet to be discovered. In addition, environmental and toxicological concerns must be addressed if additives have the potential to evaporate and redeposit themselves elsewhere.

\section{Base Oils}

Each of the base oils summarized in Table 3 has at least one significant shortcoming. The PFPE oils have short lifetimes and decay into corrosive products; the $\mathrm{HC}$ have higher vapor pressures and pour points than desired; and conventional additives are even more volatile. The siliconebased materials are poor boundary lubricants and tend to degrade into abrasive products. Newer hydrocarbon fluids such as Pennzanes ${ }^{\circledR}$ (also referred to as multiply alkylated cyclopentanes (MAC)) have significantly improved properties such as friction coefficient, lifetime, vapor pressure, and the ability to dissolve additives, and are now effectively replacing all old technology hydrocarbon fluids and some PFPE fluids. The PFPEbased fluids have heritage and therefore are still used in spite of their poorer friction coefficient, lifetime, and lack of additives. Their advantages remain their exceptionally low vapor pressures and wider useful temperature range. Ionic fluids show promise because of their extremely low vapor pressures, adequate friction coefficients, and superior lifetimes to PFPEs. A final class of oils, the silahydrocarbons, has also demonstrated useful properties such as low vapor pressures and adequate friction coefficients and lifetimes. All of the aforementioned hydrocarbon-based materials are more attractive as space lubricants because additive development theory for $\mathrm{HCs}$ is better understood than additive theory for PFPEs. 


\section{Postmortem Analysis of Space Lubricants-Fundamental Investigations}

Tribological Experiments on Base Oils and Greases

Figure 6 is a typical plot of SOT data from which important tribological information is obtained. The plots of coefficient of friction $(\mathrm{CoF})$ versus number of orbits provide a ranking of the lubricants' abilities to perform. Aside from $\mathrm{CoF}$ information, the life-number of orbits to failure (with failure defined as a $\mathrm{CoF}$ above 0.2) divided by the amount of lubricant employed in micrograms - provides a normalized lifetime used to quantitatively rank the ability of the oil to provide adequate lubrication. For a good lubricant these SOT experiments may run for several weeks, hence the need for reliably measuring smaller and smaller amounts of initial lubricant on the ball. These data provide little information regarding mode of lubricant failure, which typically progresses through several steps. During the initial phase (Region I) of the experiment the friction coefficient remains relatively constant as lubricant is consumed and during which time a "friction polymer" begins to form. Toward the end-phase of lubricant life the friction coefficient begins to rise to $>0.2$ (Region II-typically the last few hundred orbits), where degradation of the last of the original lubricant as well as the friction polymer occurs. During the final stage of the experiment (Region III) the friction coefficient rises to well over 0.3 due to metal-on-metal contact as lubricant is exhausted.

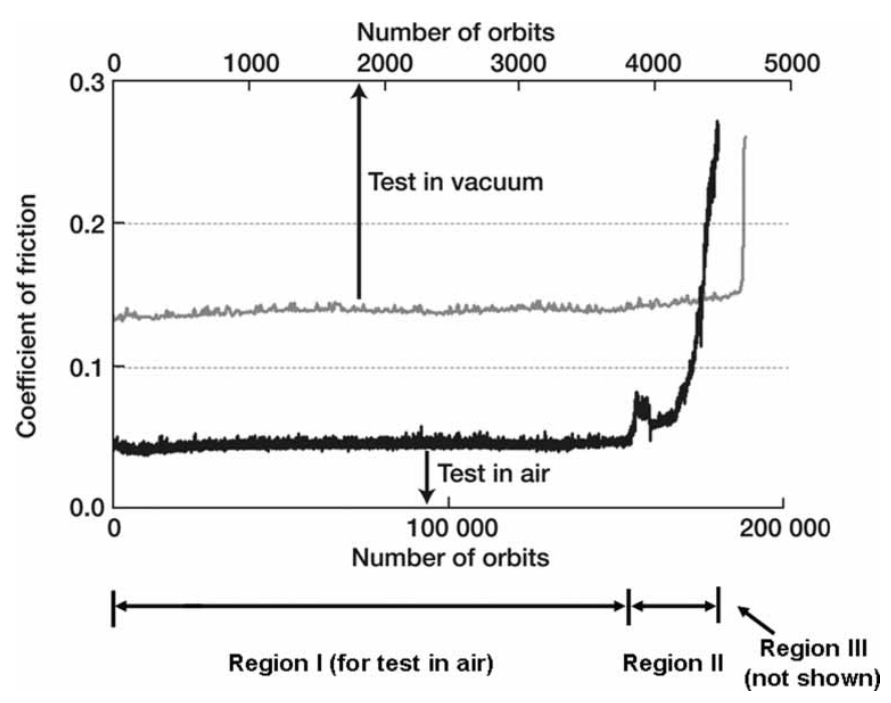

Figure 6. SOT runs for Krytox ${ }^{\mathrm{TM}} 143 \mathrm{AB}$ in air and in vacuum. 
Both midexperiment and postcomplete degradation examinations of balls and tracks in SOT experiments with PFPEs were made by various vibrational spectroscopic techniques (Herrera-Fierro et al. 2000). Due to the small track width, FTIR microscopy is routinely employed. Direct reflection off the track or crown of the ball provides adequate sensitivity for seeing any residual unused lubricant, which would exist in ridges directly adjacent to the wear track or in small patches on the surface of the ball. Our quantitative observations on patches of lubricant have consistently demonstrated little change in the IR signature (bands at 1310, 1270, 1127, and $990 \mathrm{~cm}^{-1}$ for PFPE) from Region I, indicating little structural change and that lubricant is distributed between ball and disc. In Region II, quantitative analysis of the lubricant in the track indicates near complete loss of material, and thickness varies greatly. In Region III little lubricant is left in the wear track or on the ball and the IR spectrum of the remaining brown friction polymer on both disc and ball is quite different. Broad bands in the 3600-3000, 1669, and $1436 \mathrm{~cm}^{-1}$ areas of Fig. 7 correlate well with other literature studies of polymeric fluorinated carboxylic acids (Carré and Markowitz 1985; Eapen et al. 1994; Xuan et al. 1993). The presence of a strong broad band at $3600-3000 \mathrm{~cm}^{-1}$ suggests that some of the adsorbed carboxylate is hydrogen-bonded. The strong carbonyl absorption band in dimerized carboxylic acid, typically found between 1700 to $1725 \mathrm{~cm}^{-1}$, is not apparent in the spectra. These IR spectra are indicative of sorbed metal ions; the two new peaks that appeared indicate the formation of carboxylate ion. Carboxylate ions bind to metal ions and give rise to an asymmetric stretching band between $1550-1650 \mathrm{~cm}^{-1}$ and a symmetric stretching band near $1400 \mathrm{~cm}^{-1}$ (for example see Fig. 7a). The frequencies of these two bands vary slightly depending on the metal ion involved (Nakamoto 1997; Tackett 1989).
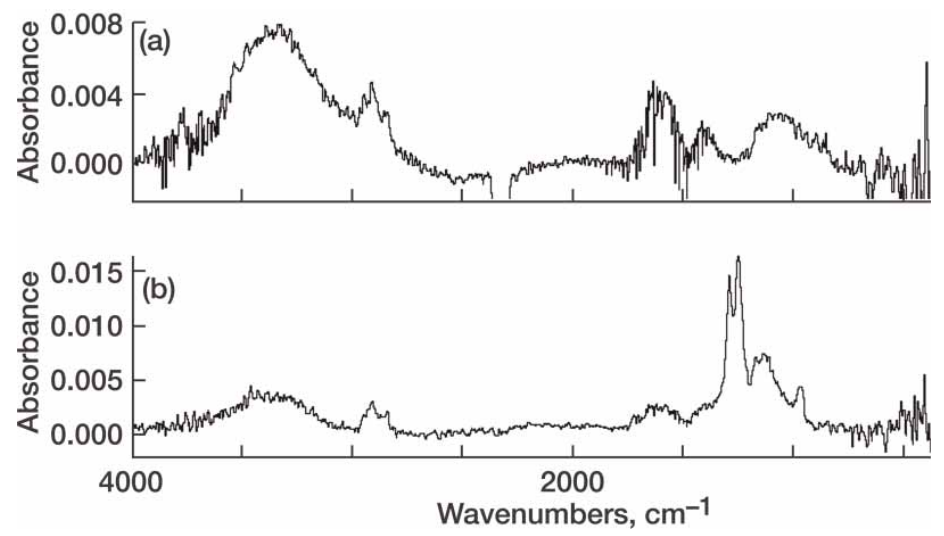

Figure 7. Infrared spectra taken from postmortem balls of SOT runs with Krytox ${ }^{\mathrm{TM}}$ $143 \mathrm{AB}$ in integration sphere: (a) ball run in air and (b) ball run in vacuum. 
Raman microspectroscopy is not often used as a quantitative technique but still provides very useful information in the postmortem analysis of oil degradation. The Raman signatures on all tribocouples tested, no matter the oil employed, are quite similar. They exhibit two extremely broad bands, often on a highly sloping luminescent background (Figs. 8d and e). All carbon materials yield unique Raman spectra, even with only subtle structural changes. Discrete molecules, such as the fullerenes, nanotubes, or nanoonions (Fig. 8a) and well-ordered $\mathrm{sp}^{3}$ material like diamond give rise to sharp spectra (unlike the broad peaks from postmortem analysis) centered at $1604 \mathrm{~cm}^{-1}$, an $E_{2 g}$ band commonly referred to as the $\mathrm{G}$ band, (and at $1364 \mathrm{~cm}^{-1}$ referred to as the disorder or D band) which are indicative of $\mathrm{sp}^{2}$ bonding in graphitized amorphous carbon (Fig. 8b) or fluorinated amorphous carbon. These spectra appear on all tribocouple surfaces, more strongly in pits and crevices in the worn metal parts.

The spectroscopic evidence led to the proposed degradation pathway for all PFPE involving defluorination, chain scission, and formation of
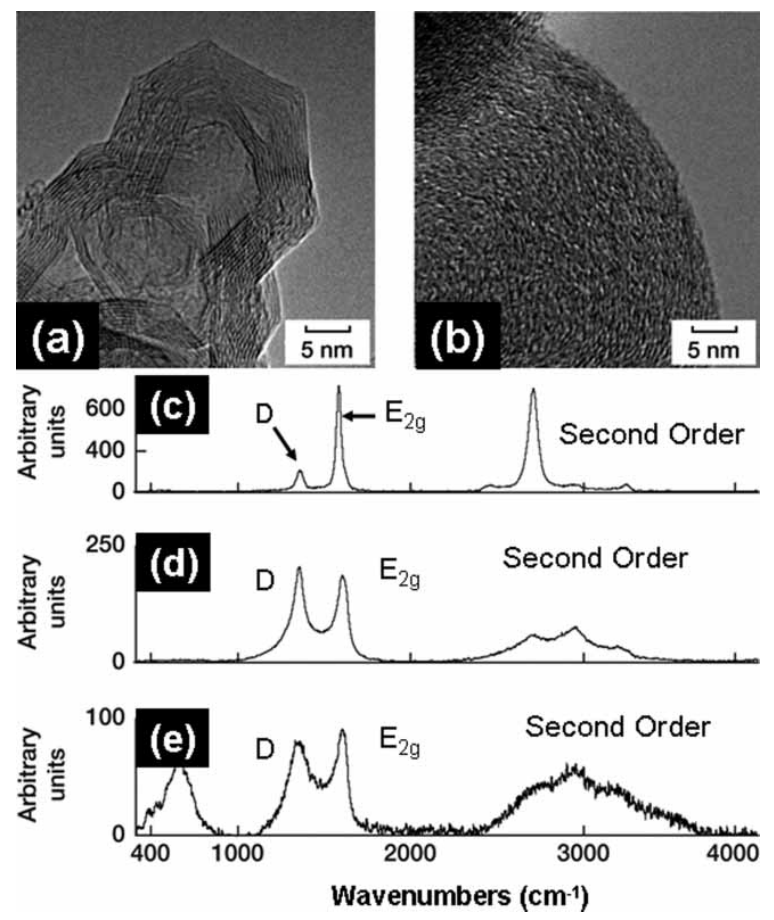

Figure 8. Nanocarbon materials and their Raman spectra: a) Carbon nano onions produced from b) carbon black. c) Raman spectrum of nano onions in (a). d) Raman spectrum of post mortem SOT ball run with Krytox ${ }^{\mathrm{TM}} 143 \mathrm{AB}$ and nano onions in vacuum and e) Raman spectrum of post mortem SOT ball run with Krytox ${ }^{\mathrm{TM}} 143 \mathrm{AB}$ and nano onions in air. 
carbonyl end groups as suggested in prior literature (Carré 1985; Eapen et al. 1994; Xuan et al. 1993). Carboxylic acid groups form quickly when carbonyl groups react with humidity in the air, and in turn react with surface metal ion species to form carboxylates. The resulting Raman spectrum arises from a fluorine-deficient network that is graphitic in nature. Neither the graphitic network nor the carboxylate species would be expected to be soluble in the Freon solvent used to wash the surfaces prior to spectroscopic analysis.

One drawback from using SOT to perform tribological investigations is the lack of wear data obtained from the rigs. The standard ASTM method D4172-94(2004)E1, adapted for ultrahigh vacuum measurements, was used to compare wear rates of various PFPE fluids (143AC, S-200, and Z-25) with three synthetic hydrocarbons, a MAC (2001a), a MAC formulated with antiwear and antioxidant additives (2001), and an unformulated PAO (PAO100), as well as an unformulated silahydrocarbon (pentasilahydrocarbon) (Jones, Poslowski et al. 1999). The wear rates determined in this investigation were ranked as follows:

$$
\begin{aligned}
& \text { PFPE } Z-25 \gg \text { PFPE S-200 } \gg \text { PFPE143AC }>\text { PAO-100 }> \\
& \text { MAC2001a }>\text { MAC2001( formulated) }>\text { pentasilahydrocarbon }
\end{aligned}
$$

The last four are within statistical insignificance of each adjacent fluid. One stationary ball from the PFPE Z-25, PFPE S-200, PFPE 143AC, and MAC 2001a runs was washed with appropriate solvent and on and off wear scars were examined by FTIR and Raman microspectroscopies. From IR spectra it is clear that the same decomposition mechanisms present in the prior investigation were present for the PFPEs in this study. Qualitatively, the quantity of the reaction products found, as determined by IR spectral intensities, correlated directly with the amount of wear for the PFPEs. The one HC lubricant examined showed little postwear material, as determined by weak peaks in the $3000-2850 \mathrm{~cm}^{-1} \mathrm{C}-\mathrm{H}$ stretch region of the spectrum. No other degradation signature was observed in the spectra for the MAC. Visual examination of balls from other lubricants showed negligible wear residue, which is why these balls were not submitted for IR analysis. All Raman spectra revealed the same signature as previously detailed, indicating that even HC oil eventually decomposed into amorphous graphitic material.

Two commercially available ionic liquids (Covalent Associates, Inc.) referred to henceforth as IL-A and IL-B (for proprietary reasons) were used for vapor pressure determinations and tribological investigations (Street and Morales et al. 2007). Ionic liquids are salts, many of which are typically viscous fluids at room temperature (characterized by negligible vapor pressures), which led to study of their effectiveness as space lubricants. Vapor pressures were determined to be on the order of the best space liquid lubricants available and consequently ionic liquids were tribologically evaluated in the SOT. Both fluids had better friction coefficients and better 
lifetimes than PFPEs, but their lifetimes were distinctly less than MACs. Postmortem analysis of balls, guide plates, and discs yielded information about the degradation mechanism for the ILs. Both ILs showed similar IR and Raman spectra for the wear areas. Broad IR peaks in the $1700-1600 \mathrm{~cm}^{-1}$ and in the $3500-3100 \mathrm{~cm}^{-1}$ regions are indicative of the build-up of carbonaceous material. This trend is observed in the final stages of lubricant lifetime, which is in line with all carbon-based lubricants examined thus far. The broad Raman peaks centered near 1360 and $1580 \mathrm{~cm}^{-1}$, the D and G peaks respectively, are indicative of amorphous graphitic materials, the final stage of carbon-based lubricants. The distinct lack of an identifiable second-order peak in the $2500-3100 \mathrm{~cm}^{-1}$ region of the Raman is another strong indicator of highly disordered graphitic material. Outside the wear areas, along the tracks in the discs and the scrub on the guide plate, droplets of unused or only slightly degraded ILs were identified from their IR signatures. The amount of shed material was not quantifiable but presumably led to lower lifetimes being reported.

Greases are lubricant base oils thickened to semisolid form with either a soluble additive, for example n-octadecylterephthalamate soap in the case of MACs, or with an inert insoluble material, PTFE Teflon in the case of PFPEs (Marchetti et al. 2002). Because greases are complex formulations, the method of lubricating the balls was changed to accommodate the presence of thickener prior to SOT testing. Grease was applied directly to a ball, which was then rolled between two plastic membranes for distribution and removal of excess grease. The membrane process was repeated until approximately the correct amount of lubricant remained on the ball. In this investigation, it was important to confirm that a representative amount of thickener, especially in the case of PTFE thickener, was applied to the balls along with base oil. Infrared spectroscopy was used to measure the ratios of oil to thickener peaks to ensure that the grease composition was approximately preserved during lubrication. The IR spectra of the decomposition products in postwear testing were similar to those described previously.

Because PFPE fluids have no soluble thickening agents, a study was undertaken to use nanostructured carbon material-carbon nano-onions shown in Fig. 8a, which we have synthesized from amorphous carbon spheres shown in Fig. 8b-as thickener. This would also act as an auxiliary lubricant in the boundary lubrication regime. The lubrication role of solid particles introduced in a fluid lubricant, generally in greases, depends on the size of particle, on the number of particles, and on the geometry of the contact. The introduction of small particles is beneficial in boundary lubrication when the ratio of film thickness to surface roughness is below 0.3 on the Stribeck lubrication curve; that is, the lubricant film is not separating the surfaces in contact. The particles present in the lubricant have to be of small size to allow good fluid circulation within the contact and to not "jam" it. The number of particles is generally low, a few percent in volume, 
and up to $10 \%$ for graphite. Under severe conditions, very small graphite particles can produce a layer that helps to protect the surfaces; however, it is the edge sites present at the graphitic lamella that promote decomposition of graphite lubricant. As an alternative to graphite we have evaluated the ability of graphitic nano-onions, $20 \%$ by weight, to make oil into a thick grease while simultaneously improving the lubricating lifetime of the oil, Krytox $^{\mathrm{TM}} 143 \mathrm{AB}$, in SOT testing (Street et al. 2004). The anticipated advantage of the nanostructured carbon is that the particles are small and contain few reactive edge sites. Tests were conducted at room temperature in ambient air and ultrahigh vacuum. Examples of friction traces and the lifetime obtained are given in Fig. 6. The use of nano-onions did not improve the lifetime, nor did it change the friction coefficient of this PFPE oil run in vacuum. However, tests in air resulted in a very low friction coefficient $(0.04-0.05)$ with a lifetime eight times greater than that of the original oil. Furthermore, the failure is more "progressive" compared to the one observed in vacuum. This is consistent with the behavior of graphite, which requires traces of water to perform effectively.

Infrared analysis was also performed postmortem on the balls (Fig. 7). Both types of balls showed peaks in the $2960-2850 \mathrm{~cm}^{-1}$ range and a broad band in the $3360 \mathrm{~cm}^{-1}$ range. These bands correspond to hydrocarbon impurities and possibly PFPE degradation products, respectively. Weak peaks in the 1600 and $1400 \mathrm{~cm}^{-1}$ range, indicative of carboxylate functional groups, were found in both spectra. The ball run in vacuum (Figs. 5 and $7 \mathrm{~b}$ ) revealed traces of material resembling the original Krytox ${ }^{\mathrm{TM}} 143 \mathrm{AB}$, having bands in the $1346-970 \mathrm{~cm}^{-1}$ region of the spectrum. This result is unexpected since all the lubricant is typically consumed during a SOT experiment. The material from Fig. 5 freely washed from the ball and 35 micrograms of Krytox ${ }^{\mathrm{TM}} 143 \mathrm{AB}$ would not have caused a failure, indicating that the material was not the original lubricant. Further interpretation of the spectra is complicated by the fact that the ratio of band intensities as a function of concentration is not a constant for the Krytox ${ }^{\mathrm{TM}}$ fluids. The observed IR spectra are a complex function of the extinction coefficient, $k$, and the refractive index, $n$. In most cases, the band intensity ratio as a function of concentration is a constant but in the case of this PFPE, the refractive index contribution to the observed spectrum causes the shape of the spectrum to vary considerably as a function of concentration (Pepper 1995). Raman analysis on the balls run in vacuum (Fig. 8d) indicates the presence of a large quantity of graphitic material having well-defined D $\left(1341 \mathrm{~cm}^{-1}\right)$ and $\mathrm{G}\left(1592 \mathrm{~cm}^{-1}\right)$ peaks as well as multiple small peaks in the second order peak region $\left(2520-3300 \mathrm{~cm}^{-1}\right)$. These spectra are not similar to those of the applied nano-onions (Fig. 8c) but resemble conventionally degraded lubricants. It should be noted that we have evidence the nano-onions degrade to produce similar spectra, so it is not possible to distinguish the source of the degraded material. The ball run in air (Fig. 8e) had similar spectral features but at much lower concentrations. Additionally, 
a moderate, broad, unidentified peak occurred at $694 \mathrm{~cm}^{-1}$ in all spectra for the balls run in air.

\section{PFPE Additive Investigations}

A major problem with the PFPEs is that they all have very short lifetimes relative to hydrocarbon-based fluids, and PFPE decomposition products, being corrosive, attack the bearing metal. Over the years considerable effort has been expended in developing soluble additives for these oils, which will improve their performance. Because these lubricants decompose into corrosive products in the boundary lubrication regime, additive efforts have focused on enhancing performance under extreme pressure. In one study (Shogrin 1999) several organic functional groups were modified with longchain pendant perfluoropolyether moieties to increase their molecular weight (lowering vapor pressure) and to promote solubility in base oils. Because only $1 \%$ additive was incorporated into the formulations, no distinguishable change in the IR spectra was observed prior to four-ball wear testing these formulations. After rinsing the wear scars with appropriate solvents, the IR and Raman peaks of degradation products previously discussed became apparent. The amount of friction polymer in the residue, as determined by the intensity of the IR spectra, directly correlated to wear as in previous investigations. In a second study (Morales 1997), a conventional silane coupling agent, 3-aminopropyltriethoxysilane, was both added to a PFPE oil and used to pretreat the tribologically contacting surfaces. The silane was not soluble in the PFPE, hence the $1 \%$ mixture was sonicated to a milky color, which was stable for two months. In space tribology, emulsions are not considered an ideal solution to the problem since the lubricated mechanism is launched into space for typically a five-year mission with no relubrication. Emulsions must be stable for the mission duration for them to be successful.

Another method for improving the lifetime of PFPE fluids is to alter the tribocouples. In these investigations, the PFPE with additive was tested against both unmodified and silane-treated tribocouples. The surface treatment produced a 3-micrometer-thick coating with IR peaks at 3367 and $3298 \mathrm{~cm}^{-1}$ (N-H antisymmetric and symmetric stretching vibrations, respectively), 2932 and $2840 \mathrm{~cm}^{-1}$ (C-H stretching vibrations), 1100 and $930 \mathrm{~cm}^{-1}$ ( $\mathrm{Si}-\mathrm{O}-\mathrm{CH}_{2}$ vibrations), and other characteristic peaks associated with this type of compound. In short duration PoD sliding experiments, the surfaces of the washed tribocouples appeared smoother and had lower wear than without silane added to the process, and the wear surface retained the IR signature of the silane treatment but did not show any signature of PFPE decomposition products. Examination of an uncoated pin run against a silane-treated disc showed the IR signature of the silane as a transfer film. In long duration experiments the wear scars lacked the IR signature of the silane but showed the signature typical of PFPE degradation products. 
Tribocouple Modification as a means of Improving PFPE Performance

As stated previously, the major degradation pathway for PFPE lubricants involves production of corrosive end products. These corrosive products attack the metal bearing parts to produce metal fluorides, which themselves are soft and lubricious but ironically serve as catalysts to accelerate further decomposition (Carré 1985; Eapen et al. 1994). In the preceding section additives were employed in attempts to react with the surface and prevent the degradation reaction. An alternate manner of accomplishing the same effect is to alter the chemistry of the tribocouples through surface alteration. Conventional surface treatments for bearings include acid passivation of the surface by chromic acid bath and phosphating by tricresyl phosphate soak, both of which have been tested to see if they would improve PFPE lubrication (Shogrin et al. 2003). Only marginal improvement was observed. In another study (Jones et al. 2000) the balls employed were coated with a thin layer of $\mathrm{TiC}$, yielding somewhat improved performance. In both of these studies IR microspectroscopy was used to examine the degradation products, which were identical to degradation products of prior studies.

\section{Postmortem Analysis of Space Lubricants-Case Studies}

In preceding sections we have mostly discussed studies performed under accelerated conditions in tribometers that simplify and therefore only simulate bearings. In order to fully understand how an actual tribological system will behave, component (i.e., actual bearing) testing is performed. In these tests more realistic conditions are employed such as using a more representative load on a bearing that is fully lubricated. Under these conditions the test can last many months to years and run through millions of cycles. One such test was performed in order to evaluate the duplex bearing pair of the Moderate Resolution Imaging Spectroradiometer (MODIS) aboard the Terra, EOS AM-1, satellite (VanDyk et al. 2002). The MODIS mission is to view Earth's entire surface, gathering data to better understand the global dynamics and processes occurring on land, in the oceans, and in the lower atmosphere. All observations are made through an extremely high resolution, optically and mechanically precise scan-mirror motor/encoder assembly. The reliable performance of this assembly depends on two duplex bearing pairs lubricated with Pennzane ${ }^{\circledR}$ SHF-X-2000, formulated with lead naphthenate. Upon disassembly, some lubricant discoloration was observed, but none of the bearings had visible damage. The bearings surfaces and oil were analyzed by a large variety of techniques including XPS, scanning electron microscopy (SEM), size exclusion chromatography (SEC), and Raman and FTIR microspectroscopy. Raman and IR analyses of the lubricant indicated the standard signature for nondegraded Pennzane ${ }^{\circledR}$. Based on the suite of analyses and because of design similarities between the engineering model 
and life test unit, and the flight scanners, it was concluded that the flight scanners had sufficient margin for the five-year mission even though SEC data indicated that some polymerization of oil had occurred.

In late 2001, a prelaunch Geostationary Operational Environmental Satellite (GOES) instrument bearing failure occurred. A prequalified bearing seized, resulting in a hold on the launch of two more units, one of which was on the launch pad in countdown at that time and could not be launched until the root cause for bearing failure was determined. The disassembled bearing was submitted to the author and IR spectroscopy was used to perform analysis of the failed bearing components. What little lubricant was detected on the balls and in the contact region of the inner race of the bearing appeared normal. From the IR data it was determined that an insufficient lubricant charge had been applied by the instrument manufacturer, resulting in premature failure, a determination that allowed timely launch of the other two properly lubricated satellites.

To give perspective on the advantage of FTIR microspectroscopy, the entire IR analysis took less than a half day. The diagnosis was later confirmed by gravimetric analysis of the residual lubricant in the bearing, a tedious process that required approximately three weeks. Extreme cost is involved with launch delays, which include maintenance of launch readiness. Costs incurred by teardown, inspection, and recertification of two satellites would be in the millions of dollars, not to mention the loss of revenue associated with failure to generate data.

Each Space Shuttle body flap contains four actuators to drive it (Street and Sovinski 2005). Four similar actuators in the tail drive the rudder/speed brake. These actuators are deemed as Critical 1 components, where failure means loss of vehicle and crew. These components are connected in series; hence failure of one actuator in a section of the craft causes complete loss of function in that section. To date, many actuators have seen service and have been disassembled for maintenance and refurbishment. Only one bearing from all the actuators examined thus far has the unique appearance of "black balls," best described as black mirrors. The received balls (both the black balls from the space shuttle Discovery body flap actuator and used balls from a similar body flap actuator on the space shuttle Atlantis) had been cleaned with Fluoroclean ${ }^{\mathrm{TM}}$ solvent. No further details were provided on the black balls and the used balls. Unfortunately, none of the extract was maintained for chemical analysis, which might have indicated degradation of the lubricant itself. These bearings were previously lubricated with grease, which contained a PFPE base oil. The IR signature of the black balls was extremely weak, possibly due to the aggressive sonication employed during the cleaning effort. Still, the classic IR signature of decomposed PFPE was evident in the $3600-3100 \mathrm{~cm}^{-1}$ range as well as at 1732 , 1534 , and $1438 \mathrm{~cm}^{-1}$. The peak at $1732 \mathrm{~cm}^{-1}$ is ascribed to unreacted carbonyls associated with the broad peak in the $3600-3100 \mathrm{~cm}^{-1}$ range, and the peaks at 1543 and $1438 \mathrm{~cm}^{-1}$ are carbonyls associated with metal 
ions. In the Raman, the classic D and G band signatures were observed. An XPS analysis of the black balls indicated the presence of iron fluoride and iron oxides. The color of iron fluoride is dependent on the oxidation state and state of hydration and ranges from white to yellow to green. Iron fluoride has been shown to catalyze the decomposition of PFPE lubricants, which could have been accelerated by high temperatures experienced by the bearing during reentry. Under normal conditions iron fluoride is stable; however, under the conditions these balls were exposed to, it is likely that the iron fluoride hydrolyzed into iron oxides and hydrogen fluoride. Some iron oxides and mixed hydroxy oxides are typically black, which may have contributed to the black appearance of the balls. From the combination of XPS, IR, and Raman spectroscopic results it was concluded that (1) this particular bearing had experienced extreme heat leading to both lubricant degradation and bearing condition and (2) this was a singular event.

\section{CONCLUSIONS}

Infrared and Raman microspectroscopies have been used to great advantage in tribological investigations, both in fundamental and actual case studies. In fundamental investigations IR spectroscopy can be used to provide quantitative information about the lubricant load employed, in tests either by microspectroscopy or by using the integration sphere technique. Qualitatively, the use of IR signature intensity has been correlated directly to wear behavior and to the elucidation of lubricant decay mechanisms. The decay mechanisms are confirmed by Raman microspectroscopy, which is complementary to IR in that IR provides useful information on functional groups attached to the lubricant carbon backbone while Raman provides information on the structure of the degraded backbone. In case studies, not only are failure mechanisms elucidated but vibrational microspectroscopic techniques allow for large parts to be easily examined intact, facilitating diagnosis.

\section{ACKNOWLEDGMENT}

The author thanks Mark Jansen for providing the figures for some of the tribometer rigs.

\section{REFERENCES}

Carré, D.J. and Markowitz, J.A. 1985. The reaction of perfluoropolyalkylether oil with $\mathrm{FeF}_{3}, \mathrm{AlF}_{3}$, and $\mathrm{AlCl}_{3}$ at elevated temperatures. ASLE Trans., 28 (1): 40-6.

Cowart, E.G. 1973. Lunar roving vehicles: spacecraft on wheels. Proc. Inst. Mech. Eng., 187: 464-91. 
de Groh, K.K., Dever, J.A., Sutter, J.K., Gaier, J.R., Gummow, J.D., Scheiman, D.A., and He, C. 2001. Thermal contributions to the Teflon ${ }^{\circledR}$ FEP on the Hubble space telescope. High Perform. Polym., 13: S401-20.

Eapen, K.C., John, P., and Liang, J.C. 1994. Degradation of a branched perfluoropolyalkylether fluid with anhydrous aluminum chloride. Macromol. Chem. Phys., 195: 2887-903.

Fusaro, R.L. and Khonsari, M.M. 1992. Liquid lubrication for space applications. NASA TM, 105198.

Gaier, J.R. 2007. The effects of lunar dust on EVA systems during Apollo missions NASA/TM2005-213610/REV1.

Glassow, F.A. 1976. Assurance of lubricant supply in wet-lubricated space bearings. Proceedings of the 10th Aerospace Mechanisms Symposium, NASA, CR-148515: 90-103.

Herrera-Fierro, P., Shogrin, B.A., and Jones, W.R., Jr. Spectroscopic analysis of perfluoropolyether lubricant degradation during boundary lubrication. Lubrication Eng., 56 (2): 23-9.

James, G.E. 1977. Positive commandable oiler for satellite bearing lubrication. 11th Aerospace Mechanisms Symposium, NASA, CP-2038: 87-95.

Jones, C.S. and Nola, F.J. 1971. Mobility systems activity for lunar rovers at MSFC NASA TM X-64623.

Jones, W.R., Jr. and Jansen, M.J. 2005. Lubrication for space applications NASA CR 213424.

Jones, W.R. Jr., Jansen, M.J., Helmick, L.H., Nguyen, Q.G., Wheeler, D.R., and Boving, H.J. 1999. The effect of stress and TiC-coated balls on lubricant lifetimes using a vacuum ball-on-plate rolling contact tribometer. NASA TM-1999-209055.

Jones, W.R. Jr., Poslowski, A.K., Shogrin, B.A., Herrera-Fierro, P., and Jansen, M.J. 1999. Evaluation of several space lubricants using a vacuum four-ball tribometer. Tribology Trans., 42 (2): 317-23.

Jones, W.R. Jr., Pepper, S.V., Wheeler, D.R., Jansen, M.J., Nguyen, Q.G., and Schröer, A. 2000. The effect of stress and TiC-coated balls on lifetime of a perfluoropolyalkylether using a vacuum rolling contact tribometer. Tribology Trans., 43 (4): $685-8$.

Jones, W.R. Jr., Toddy, T.J., Predmore, R., Shogrin, B., and Herrera-Fierro, P. 1996. The effect of ODC-free cleaning techniques on bearing lifetimes in the parched elastohydrodynamic regime. NASA TM 107322.

Katon, J.E., Sommer, A.J., and Lang, P.L. 1980. Infrared microspectroscopy. Appl. Spectrosc. Rev., 25: 173-211.

Landis, G.A. 1998. Mars dust-removal technology. J. Propulsion and Power, 14 (1): $126-8$.

Lowenthal, S.H., Scibble, H.W., Parker, R.J., and Zaretsky, E.V. 1985. Operating characteristics of a $0.87 \mathrm{~kW}$-hr flywheel energy storage module. Proceedings of the 20th Intersociety Energy Conversion Engineering Conference (IECEC), 2: $164-74$.

Lunokhod 1. 2007. http://www.aquiline-aero.com/products/greasesrus03.shtml, accessed October 30, 2007.

Lunokhod 2. 2007. http://tech-db.istc.ru/ISTC/sc.nsf/html/institutions.htm?open\& id $=1262$, accessed October 30, 2007.

Marchetti, M., Jones, W.R. Jr., Pepper, S.V., Jansen, M.J., and Predmore, R.E. 2003. In-situ, on-demand lubrication for space mechanisms. Tribology Trans., 46 (3): $452-9$. 
Marchetti, M., Jones, W.R. Jr., Street, K.W., Pepper, S.V., and Jansen, M.J. 2002. Preliminary evaluation of greases for space mechanisms using a vacuum spiral orbit Tribometer. NLGI Spokesman, 66 (2): 19-23.

Morea, S.F. 1993. The lunar roving vehicle-historical perspective. Paper presented at the 2nd Conference on Lunar Bases and Space Activities, 619-632, April, 1988, Houston, Texas.

Morales, W., Fusaro, R.L., Siebert, M., Keith, T., Jansen, R., and Herrera-Fierro, P. 1997. A new antiwear additive/surface pretreatment for PFPE liquid lubricants. Tribology Trans., 40 (2): 321-9.

Nakamoto, K. 1997. Infrared and Raman Spectroscopy of Inorganic and Coordination Compounds, Part B, 5th edn.; John Wiley \& Sons: New York.

Nye Lubricants, Inc. 2007. Fairhaven, MA. Available through: http://www. nyelubricants.com/index.htm, accessed October 30, 2007.

Pepper, S.V. 1995. Characterization and application of a grazing angle objective for quantitative infrared reflection microscopy. Appl. Spectrosc., 49 (3): 354-60.

Pepper, S.V. 2005. Effect of test environment on lifetime of a vacuum lubricant, Proceedings of World Tribology Congress III, Abstract WTC2005-63982. Washington, D.C.

Shogrin, B.A., Jones, W.R. Jr., and Herrera-Fierro, P. 1996. Spontaneous dewetting of a perfluoropolyether. Lubrication Eng., 52 (9): 712-18.

Shogrin, B.A., Jones, W.R. Jr., Herrera-Fierro, P., and Jansen, M.J. 2003. The effects of acid passivation, tricresyl phosphate presoak, and UV/ozone treatment on the tribology of perfluoropolyether-lubricated $440 \mathrm{C}$ stainless steel couples. J. Synthetic Lubrication, 19 (4): 283-302.

Shogrin, B.A., Jones, W.R. Jr., Herrera-Fierro, P., Lin, T-Y., and Kawa, H. 1999. Evaluation of boundary enhancement additives for perfluoropolyethers. Tribology Trans., 42 (4): 747-54.

Street, K.W., Marchetti, M., Vander Wal, R.L., and Tomasek, A.J. 2004. Evaluation of the tribological behavior of nano-onions in Krytox 143AB. Tribol. Lett., 16 (1-2): $143-9$.

Street, K.W. Jr., Morales, W., and Koch, V.R. 2007. Evaluation of vapor pressure and ultra-high vacuum tribological properties of ionic liquids. Proceedings of the Society of Tribologists and Lubrication Engineers 62nd Annual Meeting. Philadelphia, Pennsylvania.

Street, K.W., Pepper, S.V., Wright, A., and Grady, B. 2007. Characterization of lubricants on ball bearings by FTIR using an integrating sphere. Tribotest J., 13 (3): 129-37.

Street, K.W. and Sovinski, M.F. 2005. Analysis of black bearing balls from a space shuttle body flap actuator. Proceedings of World Tribology Congress III, Abstract WTC2005-63539. Washington, D.C.

Tackett, J.E. 1989. FTIR characterization of metal acetates in aqueous solution. Appl. Spectrosc., 43 (3): 483-9.

VanDyk, S.G., Dietz, B.J., Street, K.W., Jones, W.R. Jr., Jansen, M.J., Dube, M.J., Sharma, R.K., and Predmore, R.E. 2002. The role of bearing and scan mechanism life testing in flight qualification of the MODIS instrument. Tribotest J., 9 (2): $139-56$.

Xuan, J., Chen, G.-L., and Chao, J. 1993. Organic buildup on slider leading edge tapers and its effect on wet stiction. IEEE Trans. on Magnetics, 29: 3948-50. 